\title{
CTHRC1 mediates IL-1 $\beta$-induced apoptosis in chondrocytes via JNK1/2 signaling
}

\author{
QI ZHANG, ZONG-SHENG YIN, FU-WEN ZHANG, KUN CAO and HE-YAN SUN
}

\begin{abstract}
Department of Orthopedics, The First Affiliated Hospital of Anhui Medical University, Hefei, Anhui 230022, P.R. China
\end{abstract}
Received May 18, 2017; Accepted January 8, 2018

DOI: $10.3892 / \mathrm{ijmm} .2018 .3403$

\begin{abstract}
Osteoarthritis (OA), also known as degenerative joint disease or degenerative arthritis, is characterized by chondrocyte apoptosis. The aim of the present study was to investigate the effects of collagen triple helix repeat containing 1 (CTHRC1) and the c-Jun N-terminal kinase (JNK) 1/2 inhibitor SP600125 on rat chondrocytes cultured in vitro with interleukin (IL)-1 $\beta$. Chondrocytes were treated with different doses of IL-1 $\beta$ and cell viability and CTHRC1 expression were assessed using Cell Counting Kit- 8 and western blot assays, respectively. In separate experiments, chondrocytes were treated with CTHRC1-expressing constructs (pLVX-Puro-CTHRC1) and/or SP600125, or IL-1 $\beta$ with either CTHRC1 short hairpin (sh)RNA constructs (shNRA-CTHRC1) or SP600125. The expression of CTHRC1, B-cell lymphoma (Bcl)-2, Bcl-2-associated X protein (Bax), cleaved caspase-3, poly ADP ribose polymerase (PARP)-1 and matrix metalloproteinase (MMP)-13 was measured using reverse transcription-quantitative polymerase chain reaction and western blotting assays. A Cell Counting Kit-8 assay was performed to examine cell viability. Annexin V/propidium iodide staining and flow cytometry assays were used to detect chondrocyte apoptosis. The expression of JNK1/2 and phosphorylated JNK1/2 was measured using western blotting. CTHRC1 was highly expressed in patients with OA compared with normal controls. IL-1 $\beta$ treatment $(5,10$ and $20 \mathrm{ng} / \mathrm{ml})$ increased the protein expression of CTHRC1 in a dose-dependent manner and decreased the viability of chondrocytes in a time-dependent manner. pLVX-Puro-CTHRC1 mimics the effect of IL-1 $\beta$ on chondrocyte apoptosis and JNK1/2 activity, and this is reversed by SP600125 treatment. However, transfection with shRNA-CTHRC1 or treatment with SP600125 inhibited IL-1 $\beta$-induced cell apoptosis and JNK1/2 activation. These results indicate that CTHRC1 downregulation may
\end{abstract}

Correspondence to: Dr Zong-Sheng Yin, Department of Orthopedics, The First Affiliated Hospital of Anhui Medical University, 218 Jixi Road, Hefei, Anhui 230022, P.R. China E-mail: yinzs2016@163.com

Key words: chondrocyte, interleukin-1 $\beta$, collagen triple helix repeat containing 1 , apoptosis, c-Jun $\mathrm{N}$-terminal kinase $1 / 2$ protect chondrocytes from IL-1 $\beta$-induced apoptosis by inactivating the $\mathrm{JNK} 1 / 2$ pathway.

\section{Introduction}

Osteoarthritis (OA) is a prevalent degenerative joint disease associated with aging, obesity and trauma and is the most common form of arthritis characterized by the progressive destruction of articular cartilage, with $>270$ million cases reported worldwide (1). Approximately $80 \%$ of OA cases diagnosed by radiography occur in patients over 65 years old (2). OA typically causes joint instability, pain, loss of function, stiffness and mobility difficulties (3), altogether leading to a deterioration in quality of life and increasing the cost of health care for the aging population (4). The etiology and pathogenesis of OA are not well understood due to a combination of various risk factors and initiating mechanisms (5). Furthermore, a significant and positive correlation between the degeneration of articular cartilage (6), which is the root cause of $\mathrm{OA}$, and bioactive compounds, including pro-inflammatory cytokines and a dipokines (7), has been demonstrated.

Although the pathogenesis of OA has multiple underlying mechanisms that are not well understood, it has been reported that decreased chondrocyte proliferation and apoptosis dysregulation are significantly associated with OA pathogenesis and are observed in OA cartilage more frequently compared with normal cartilage (8). Furthermore, chondrocyte apoptosis is positively correlated with cartilage degradation during the development and progression of OA (9). Chondrocytes are required to maintain cartilage structure and function via the production of extracellular matrix components (10), which are responsible for maintaining the cartilaginous matrix. Consequently, the coordinated regulation of chondrocyte proliferation and apoptosis is of great importance in cartilage function and cartilage injury repair due to OA. Pro-inflammatory cytokines, including interleukin- $1 \beta$ (IL-1 $\beta$ ), are important for dysregulated chondrocyte apoptosis and, together with matrix metalloproteinases (MMPs), trigger a vigorous pro-inflammatory response $(11,12)$. In addition to MMP-1, MMP-13 is also important in the pathological process of $\mathrm{OA}$, inducing matrix degradation, further chondrocyte senescence and aging changes (13). However, cellular responses to upregulation and downregulation of IL-1 $\beta$ do not dominate the overall gene expression signature 
in osteoarthritic chondrocytes (14). Therefore, the mechanism of IL-1 $\beta$ regulation in osteoarthritic cartilage degeneration remains unclear.

A previous study reported that the expression of collagen triple helix repeat containing 1 (CTHRC1) was upregulated in OA (15), indicating a central role of CTHRC1 in OA progression. However, the molecular mechanisms of CTHRC1 associated with the development and progression of OA are not well understood. The aim of the present study was to investigate the function of CTHRC1 in an IL-1 $\beta$-induced OA model in rat chondrocytes in vitro. The results suggest that CTHRC1 downregulation inhibits IL-1 $\beta$-induced chondrocyte apoptosis via inactivating JNK1/2 signaling.

\section{Materials and methods}

Tissue specimens. OA joint fluid samples $(\mathrm{n}=50)$ were collected from patients $(67.9 \pm 7.2$ years; male: female, 11:39) with OA who underwent knee arthroplasty at the First Affiliated Hospital of Anhui Medical University (Hefei, China) between June 2012 and April 2016. Human normal joint fluid samples were collected from 30 patients $(62.8 \pm 11.2$ years old; male: female, 1:4) with trauma and no history of OA or other joint diseases at The First Affiliated Hospital of Anhui Medical University. Patients who presented with obvious joint injury or with generalized OA were excluded from the study. The present study was approved by the Ethics Committee of Anhui Medical University. Written informed consent was obtained from all participants of this study and all investigations were performed in accordance with the Declaration of Helsinki. All patients agreed to the use of their samples in scientific research.

Cell culture. Articular chondrocytes were harvested from 20 male 4-week-old Sprague Dawley rats (250-300 g; Shanghai BK Experimental Animal Center, Shanghai, China), which were provided with free access to food and water and kept under a $12 \mathrm{~h} \mathrm{light/dark} \mathrm{cycle} \mathrm{at} \mathrm{a} \mathrm{constant} \mathrm{temperature} \mathrm{of} 25^{\circ} \mathrm{C}$ in a humidified atmosphere conaining $5 \% \mathrm{CO}_{2}$. Chondrocytes were treated with $75 \%$ ethanol for 10 min, washed with PBS and digested with $4 \mathrm{ml}$ collagen II (EMD Millipore, Billerica, MA, USA) at $37^{\circ} \mathrm{C}$ for $5 \mathrm{~h}$. The chondrocytes were collected by centrifugation at $400 \mathrm{x}$ g for $5 \mathrm{~min}$ at $37^{\circ} \mathrm{C}$ and resuspended with Dulbecco's modified Eagle's medium (DMEM; Gibco; Thermo Fisher Scientific, Inc., Waltham, MA, USA) containing $15 \%$ fetal bovine serum (FBS; HyClone; Thermo Fisher Scientific, Inc., Logan, UT, USA) and cultured at $37^{\circ} \mathrm{C}$ in an atmosphere containing $5 \% \mathrm{CO}_{2}$. Immunohistochemistry was performed when cells reached $50-60 \%$ confluence and cells were subsequently cultured in DMEM supplemented with $10 \% \mathrm{FBS}$ and $1 \%$ penicillin/streptomycin at $37^{\circ} \mathrm{C}$ in an atmosphere containing $5 \% \mathrm{CO}_{2}$. The present study was approved by the Ethics Committee of Anhui Medical University.

Construction of pLKO.1-CTHRC1-short hairpin (sh)RNA lentiviral vector and transfection. The specific shRNA sequence that targets the CTHRC1 coding sequence was used, with a scramble shRNA sequence used as negative control. shRNAs (40 nM) were cloned into the pLKO.1 lentiviral vector
(Addgene, Inc., Cambridge, MA, USA). The sequence of target and scramble shRNA were as follows: 5'-ATCCCAAGTATA ATGGGAT-3'; 5'-ATCTGGAGAGATCCAATAT-3'. A total of 1,000 ng pLKO.1, $900 \mathrm{ng}$ psPAX2 and $100 \mathrm{ng}$ pMD2G (all Addgene, Inc.) were then co-transfected into 293T cells (American Type Culture Collection, Manassas, VA, USA). Chondrocytes were transfected with pLKO.1-CTHRC1-shRNA (shNRA-CTHRC1) using Lipofectamine 2000 (Invitrogen; Thermo Fisher Scientific, Inc.) according to the manufacturer's protocol. The cells were analyzed $48 \mathrm{~h}$ following transfection.

Construction of pLVX-Puro-CTHRC1 lentiviral vector and transfection. cDNA encoding CTHRC1 was obtained using GENEWIZ (Suzhou, China) and cloned into pLVX-Puro to generate a CTHRC1 expression vector. The forward primer was 5'-GCGAATTCATGCACCCCCAAGGCCGCG-3' and the reverse primer was 5'-CGGGATCCTTATTTTGGTAGTTCT TCAAT-3'. A total of 1,000 ng pLVX-Puro $900 \mathrm{ng}$ psPAX2 and $100 \mathrm{ng}$ pMD2G were then co-transfected into 293T cells. Chondrocytes were transfected with pLVX-Puro-CTHRC1 using Lipofectamine 2000 according to the manufacturer's protocol. The empty pLVX-Puro vector was used as control. The cells were analyzed $48 \mathrm{~h}$ following transfection.

Cell proliferation assay. The proliferation of chondrocytes was measured using a Cell Counting Kit-8 (CCK-8) assay. Briefly, the chondrocytes $\left(3 \times 10^{3}\right.$ cells/well) were cultured with at $37^{\circ} \mathrm{C}$ overnight in an atmosphere containing $5 \% \mathrm{CO}_{2}$, following which they were treated with $0,5,10$ or $20 \mathrm{ng} / \mathrm{ml}$ IL-1 $\beta$ for $0,24,48$ and $72 \mathrm{~h}$ and incubated with $10 \mu \mathrm{l} \mathrm{CCK}-8$ solution at $37^{\circ} \mathrm{C}$ for $1 \mathrm{~h}$ in an atmosphere containing $5 \% \mathrm{CO}_{2}$. Cell proliferation was calculated using a microplate reader (ELX 800; Bio-Tek Instruments, Inc., Winooski, VT, USA) at a wavelength of $450 \mathrm{~nm}$.

Cell apoptosis assay. The apoptotic rate was evaluated using an Annexin V-fluorescein isothiocyanate (FITC) apoptosis detection kit (Beyotime Institute of Biotechnology, Haimen, China). Briefly, the chondrocytes $\left(5 \times 10^{4}\right.$ cells/well) were centrifuged at $1,000 \mathrm{x}$ g for $5 \mathrm{~min}$ at $4^{\circ} \mathrm{C}$. Pellets were fixed overnight in $70 \%$ cold ethanol. Following fixation, cells were washed twice with PBS and incubated in PBS containing RNase $(1 \mathrm{mg} / \mathrm{ml})$ for $10 \mathrm{~min}$ at room temperature. Finally, samples were mixed with $195 \mu \mathrm{l}$ Annexin V FITC and $5 \mu \mathrm{l}$ propidium iodide and incubated for $15 \mathrm{~min}$ at $4^{\circ} \mathrm{C}$. The apoptotic cells were analyzed by flow cytometry (BD Accuri C6; software version 1.0.264.21; BD Biosciences, San Jose, CA, USA).

Assay of caspase-3 activity. The caspase-3 colorimetric assay kit (KGA203; Kaiji Biological Engineering Materials Co., Ltd., Nanjing, China) was used according to the manufacturer's protocol to examine the activity of caspase-3. Chondrocytes $\left(5 \times 10^{6}\right.$ cells $\left./ \mathrm{ml}\right)$ were collected by centrifugation at $1,000 \times \mathrm{g}$ for 5 min at $4^{\circ} \mathrm{C}$, resuspended in $150 \mu \mathrm{l}$ of chilled cell lysis buffer (KGA203; Kaiji Biological Engineering Materials Co., Ltd.) and incubated on ice for $1 \mathrm{~h}$, during which cells were shocked (220 V) 3-4 times every $10 \mathrm{sec}$. Following centrifugation at $400 \mathrm{x} \mathrm{g}$ for $1 \mathrm{~min}$ at $4^{\circ} \mathrm{C}$, the supernatant was transferred to a fresh tube and $50 \mu \mathrm{l}$ of cell lysis buffer containing 100-200 $\mu \mathrm{g}$ protein was added with $50 \mu \mathrm{l} 2 \mathrm{X}$ Reaction Buffer and $5 \mu \mathrm{l}$ 
caspase- 3 substrate and incubated at $37^{\circ} \mathrm{C}$ for $4 \mathrm{~h}$ in the dark. Samples were read at $405 \mathrm{~nm}$ using a Multiskan EX microplate reader (Labsystems, Helsinki, Finland).

RT-quantitative PCR. Total RNA was extracted using TRIzol (Invitrogen; Thermo Fisher Scientific, Inc.) according to the manufacturer's protocol. cDNA was synthesized from isolated RNA using a PrimeScript RT reagents kit (Takara, Dalian, China). The conditions were as follows: $37^{\circ} \mathrm{C}$ for $60 \mathrm{~min}$, $85^{\circ} \mathrm{C}$ for $5 \mathrm{~min}$ and $4^{\circ} \mathrm{C}$ for $5 \mathrm{~min}$. PCR was performed using a DyNAmo Flash SYBR Green qPCR kit (Finnzymes Oy; Thermo Fisher Scientific, Inc.). The PCR cycling conditions were as follows: $95^{\circ} \mathrm{C}$ for $10 \mathrm{~min}$, followed by 40 cycles at $95^{\circ} \mathrm{C}$ for $15 \mathrm{sec}$ and $60^{\circ} \mathrm{C}$ for $45 \mathrm{sec}$, a final extension step of $95^{\circ} \mathrm{C}$ for $15 \mathrm{sec}, 60^{\circ} \mathrm{C}$ for $1 \mathrm{~min}, 95^{\circ} \mathrm{C}$ for $15 \mathrm{sec}$ and $60^{\circ} \mathrm{C}$ for $15 \mathrm{sec}$. Data collection was performed using an Applied Biosystems 7300 Fast Real-Time PCR System (Thermo Fisher Scientific, Inc.) and relative quantification of gene expression was calculated using the $2^{-\Delta \Delta C q}$ method (16) with GAPDH as a reference gene. To compare relative mRNA expression levels, the expression of CTHRC1, B-cell lymphoma (Bcl)-2, Bcl-2-associated X protein (Bax), cleaved caspase-3, poly ADP ribose polymerase (PARP)-1 and matrix metalloproteinase (MMP)-13 were given as ratios to GAPDH. The primers were designed using Primer Express software (v3.0.1; Thermo Fisher Scientific, Inc.) and were as follows: CTHRC1, forward 5'-CTCGCTTCGGCTCAAATG-3' and reverse 5'-GCACCA ATCCCTTCACAG-3'; MMP-13, forward 5'-CAGACAGCA AGAATAAAGAC-3' and reverse 5'-CAACATAAGCACAGT GTAAC-3'; Bcl-2, forward 5'-GGGATGCCTTTGTGGAAC-3' and reverse 5'-GTCTGCTGACCTCACTTG-3'; Bax, forward 5'-GGACGCATCCACCAAGAAG-3' and reverse 5'-CTG CCACACGGAAGAAGAC-3'; caspase-3, forward 5'-GGC ATCTCCTGTGATTGG-3' and reverse 5'-CTCAGCACTCTG GGAAAG-3'; GAPDH, forward 5'-GGAGTCTACTGGCGT CTTCAC-3' and reverse 5'-ATGAGCCCTTCCACGATGC-3'.

Western blotting. Total protein was extracted in lysis buffer supplemented with protease inhibitors (Beyotime Institute of Biotechnology). A total of $15 \mu \mathrm{l} /$ lane protein was separated by $10-15 \%$ SDS-PAGE and electrophoretically transferred onto polyvinylidene fluoride membranes. The membranes were incubated with primary antibodies: Anti-CTHR 1 (1:1,000 dilution; cat. no. 16534-1-AP; ProteinTech Group, Inc., Chicago, IL, USA), anti-p-JNK1/2 (1:2,000 dilution; cat. no. 9255; Cell Signaling Technology, Inc., Danvers, MA, USA), anti-JNK1/2 (1:1,000 dilution; cat. no. 9252; Cell Signaling Technology, Inc.), anti-Bax (1:300 dilution; cat. no. Sc-493; Santa Cruz Biotechnology, Inc., Dallas, TX, USA), anti-Bcl-2 (1:300 dilution; cat. no. Sc-492; Santa Cruz Biotechnology, Inc.), anti-Caspase-3 (1:500 dilution; cat. no. ab44976, Abcam, Cambridge, MA, USA), anti-PARP1 (cat. no. ab32064, Abcam), anti-MMP-13 (1:3,000 dilution; cat. no. ab39012; Abcam), and anti-GAPDH (1:200 dilution; cat. no. 5174; Cell Signaling Technology, Inc.) overnight at $4^{\circ} \mathrm{C}$, and subsequently incubated with a horseradish peroxidase-coupled secondary antibody, following which they were detected using enhanced chemiluminescence (cat. no. WBKLS0100; EMD Millipore) according to the manufacturer's instructions (Pierce; Thermo Fisher Scientific, Inc.).
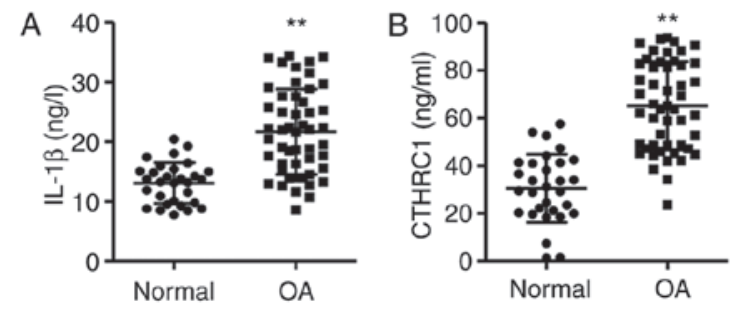

Figure 1. CTHRC1 expression is increased in human OA compared with normal tissues. (A) IL-1 $\beta$ and (B) CTHRC1 levels in the joint fluid of patients with $\mathrm{OA}$ and control patients were assessed using ELISA. ${ }^{* *} \mathrm{P}<0.01$ vs. normal. CTHRC1, collagen triple helix repeat containing 1; OA, osteoarthritis; IL, interleukin.

Immunohistochemistry. Cells were washed with $0.02 \mathrm{M}$ PBS, fixed with $4 \%$ methanol for $30 \mathrm{~min}$ at room temperature, incubated with $3 \% \mathrm{H}_{2} \mathrm{O}_{2}$ for $10 \mathrm{~min}$ and washed three times with $0.02 \mathrm{M}$ PBS. Slides were incubated with anti-Collagen II (cat. no. ab34712; 1:200 dilution; Abcam) or anti-Sry-type high mobility group-box (SOX)9 (cat. no. ab185230; 1:200 dilution; Abcam) antibody at $4{ }^{\circ} \mathrm{C}$ overnight and subsequently washed three times with $0.02 \mathrm{M}$ PBS. The slides were stained with horseradish peroxidase-labeled goat anti-rat immunoglobulin G (cat. no. D-3004; 1:500 dilution; Shanghai Long Island Biotec Co., Ltd., Shanghai, China) for $30 \mathrm{~min}$ at $37^{\circ} \mathrm{C}$ and washed three times in PBS for 3 min each time. Subsequently, the sections were stained with diaminobenzidine for $5 \mathrm{~min}$ at room temperature, counterstained with hematoxylin for $3 \mathrm{~min}$ at room temperature and washed in water. For the negative controls, the primary antibody was omitted. Images were captured using a light microscope (Olympus Corporation, Tokyo, Japan; magnification, x200).

ELISA. IL-1 $\beta$ and CTHRC1 expression in the joint fluid of patients with OA was determined using CTHRC1 (cat. no. CSB-EL006162HU; Cusabio Biotech Co., Ltd., College Park, MD, USA) and IL-1 3 (cat. no. 583311; Cayman Chemical Company, Ann Arbor, MI, USA) ELISA kits according to the manufacturer's protocol.

Statistical analysis. Results are presented as the mean \pm standard deviation. All data were analyzed using SPSS 18.0 software (SPSS, Inc., Chicago, IL, USA). Comparisons were made using t-test, analysis of variance and post-hoc test. $\mathrm{P}<0.05$ was considered to indicate a statistically significant difference.

\section{Results}

IL-1 $\beta$ and CTHRCl are upregulated in patients with OA. Joint fluid from patients with OA was assessed using ELISA kits. The results demonstrated that the levels of IL-1 $\beta$ and CTHRC1 were significantly higher in the joint fluid of patients with $\mathrm{OA}$ compared with the normal controls, with IL-1 $\beta$ and CTHRC1 expression 65.7 and $113.6 \%$ higher, respectively ( $\mathrm{P}<0.01$; Fig. 1).

CTHRCl expression is increased in IL-1 $\beta$-induced rat chondrocytes. To investigate the role of CTHRC1 in osteoarthritic chondrocytes in vitro, primary rat chondrocytes were collected and chondrocyte-asociated genes were assessed 

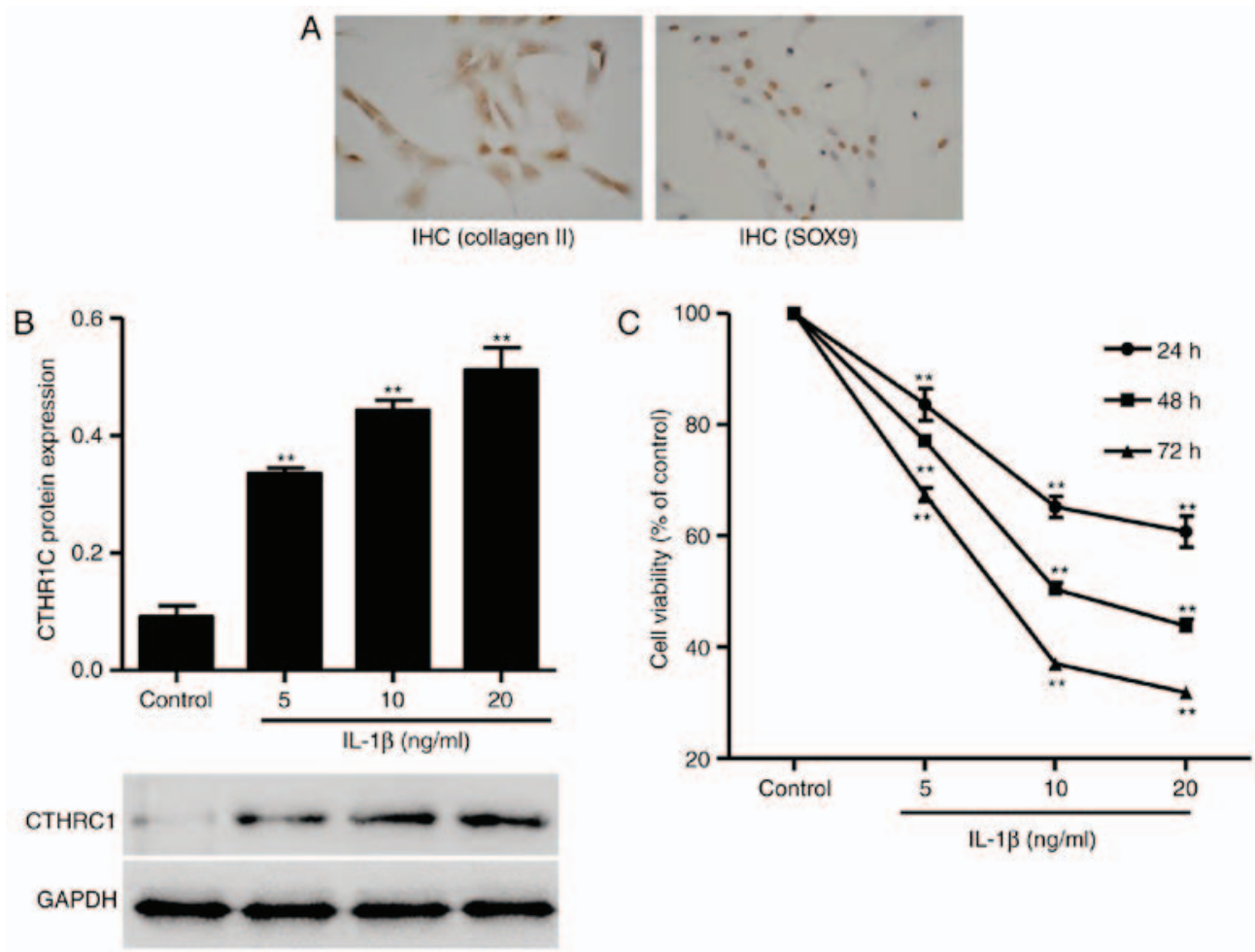

Figure 2. IL-1 $\beta$ increases CTHRC1 expression and inhibits chondrocyte proliferation. (A) Chondrocytes were collected from rat articular cartilage and the expression of Collagen II and SOX9 was measured using IHC. (B) Rat chondrocytes were treated with 5, $10 \mathrm{and} 20 \mathrm{ng} / \mathrm{ml}$ IL- $1 \beta$ and the expression of CTHRC1 was assessed using western blotting. (C) Cell proliferation was measured using a CCK-8 assay. ${ }^{* *} \mathrm{P}<0.01$ vs. control. IL, interleukin; CTHRC1, collagen triple helix repeat containing 1; SOX9, Sry-type high mobility group-box; IHC, immunohistochemistry; CCK-8, Cell Counting Kit-8.
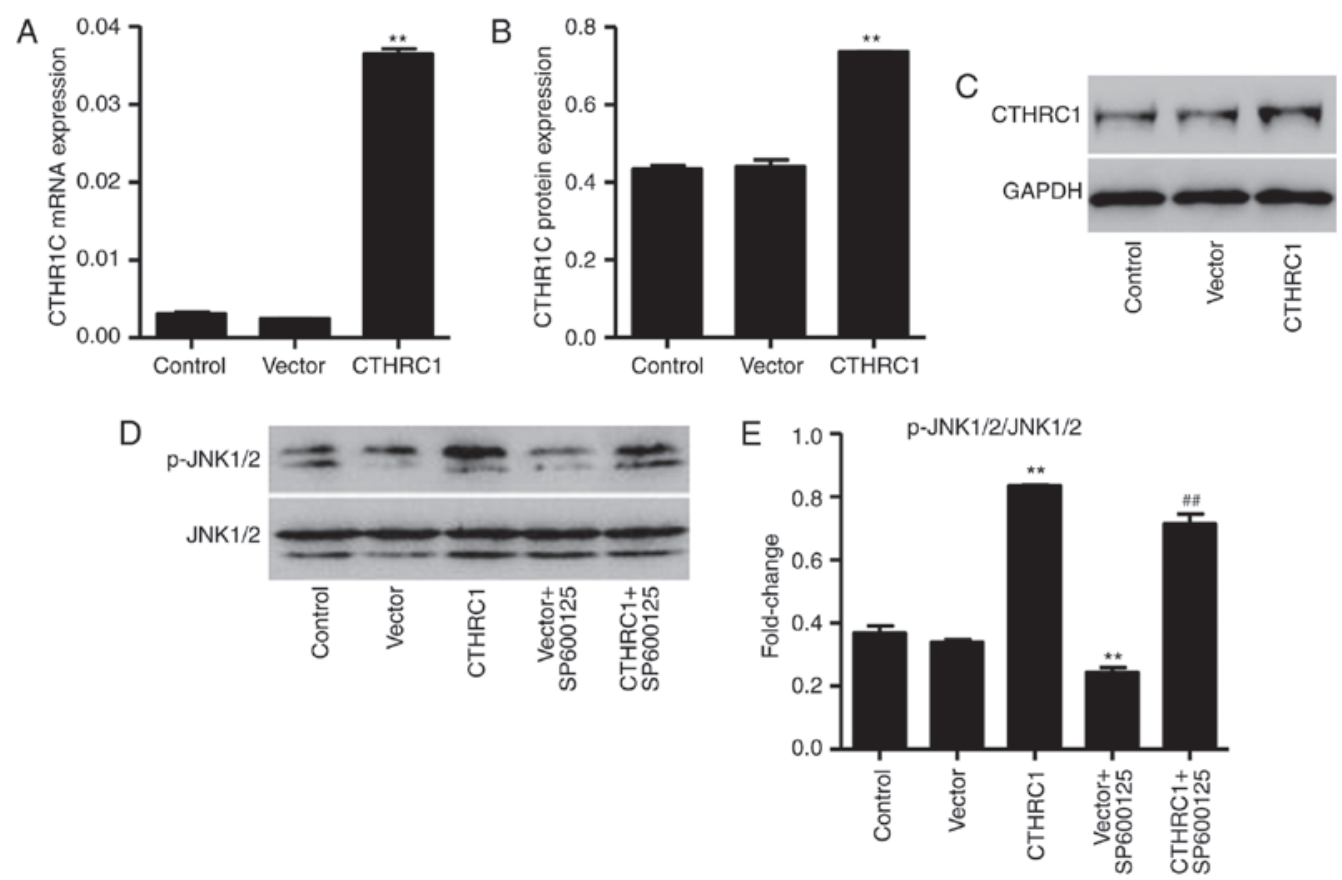

Figure 3. CTHRC1 upregulation increases JNK1/2 activation. IL-1 $\beta$-induced chondrocytes were transfected with pLVX-Puro-CTHRC1 for $24 \mathrm{~h}$ and CTHRC1 expression was assessed using (A) reverse transcription-quantitative polymerase chain reaction and (B and C) western blotting. The expression of p-JNK1/1 and JNK1/2 in IL-1 $\beta$-induced chondrocytes following pLVX-Puro-CTHRC1 transfection or SP600125 treatment was assessed using (D) western blotting and (E) quantified. ${ }^{* *} \mathrm{P}<0.01$ vs. vector and ${ }^{\# \#} \mathrm{P}<0.01$ vs. pLVX-Puro-CTHRC1. CTHRC1, collagen triple helix repeat containing 1 ; JNK, c-Jun N-terminal kinase; IL, interleukin; p, phosphorylated.

using immunohistochemistry. SOX9 is a member of the Sox gene family, which are predominantly expressed in cartilage and activate Collagen II (17). Immunohistochemical staining demonstrated that Collagen II and SOX9 were highly expressed in normal primary cultured chondrocytes (Fig. 2A), which was indicative of a well-established chondrocyte system. 

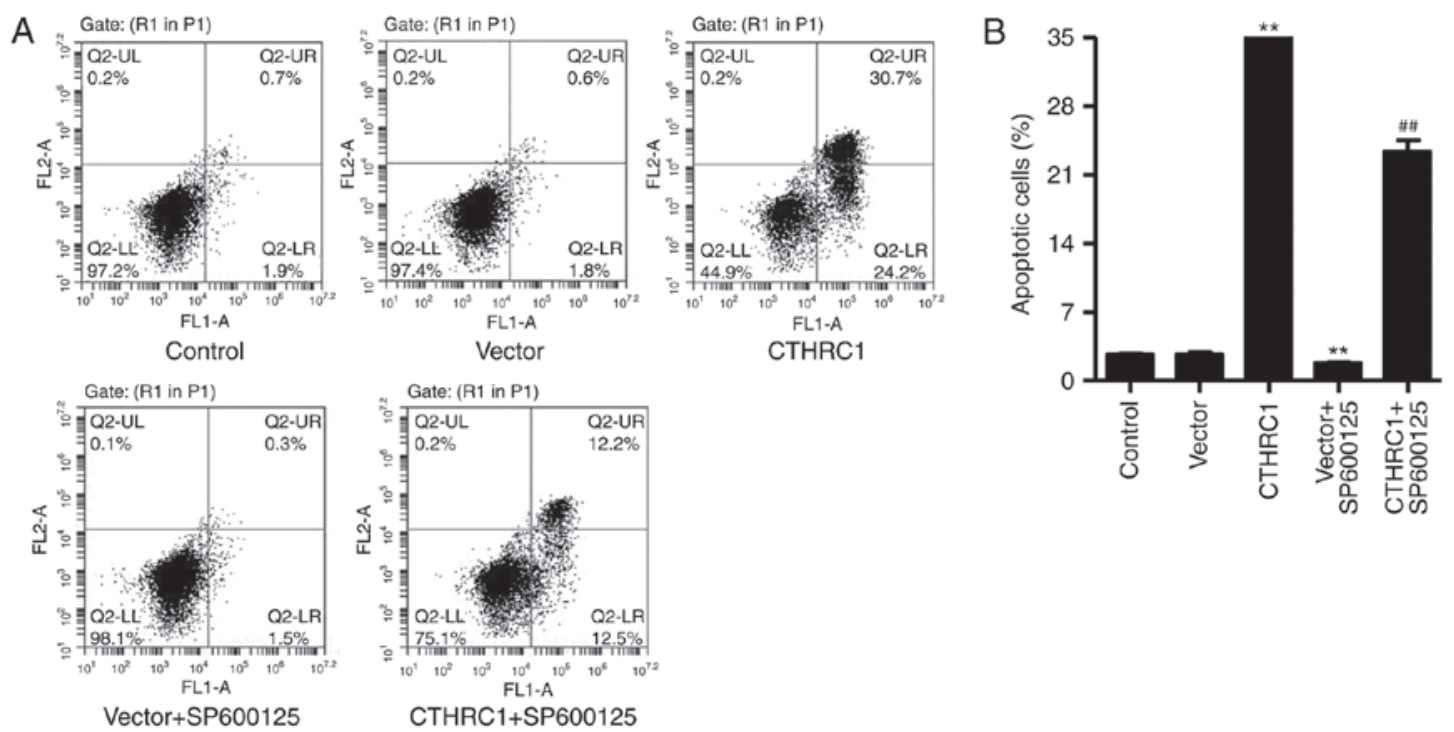

CTHRC

CTHRC1+SP600125
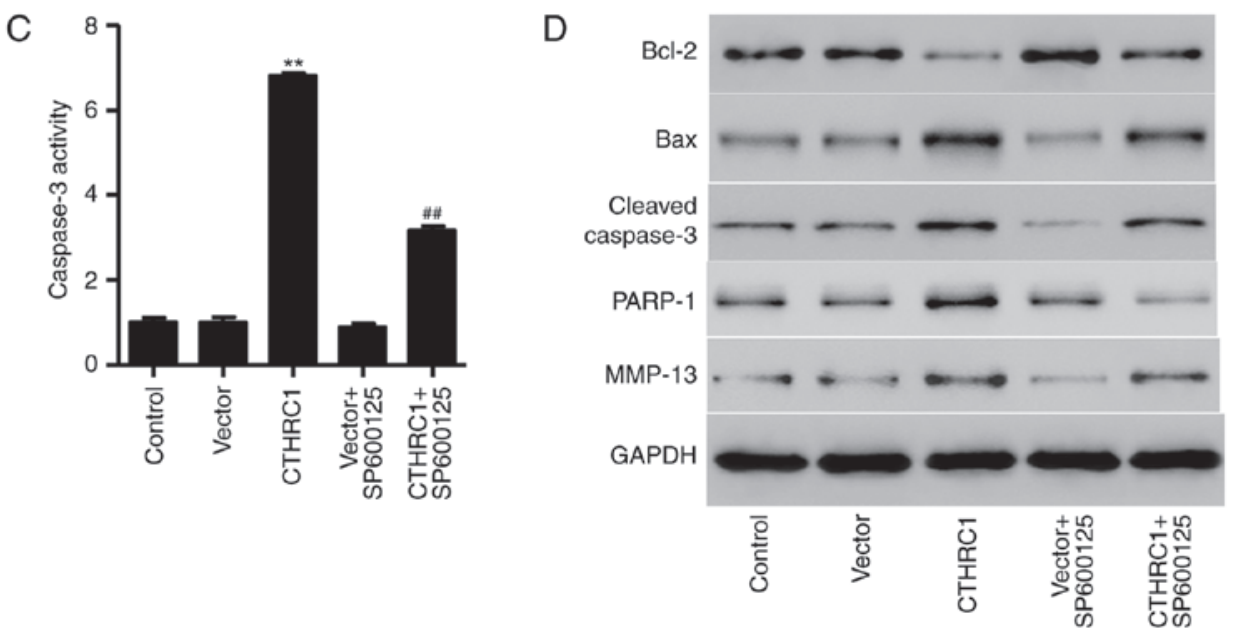

$\mathrm{E}$

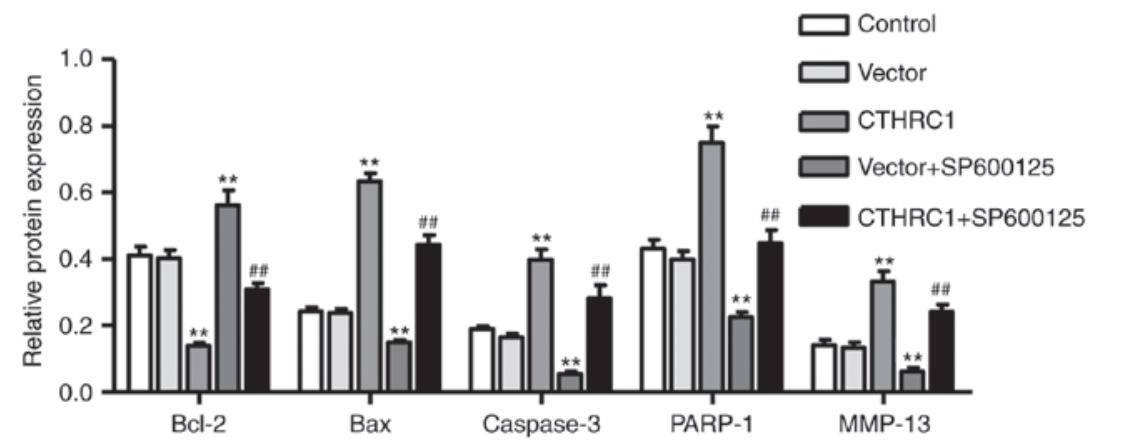

Figure 4. CTHRC1 upregulation induces chondrocyte apoptosis. (A and B) Chondrocyte apoptosis was assessed by flow cytometry analysis. (C) Changes in caspase-3 activity were investigated using spectrophotometry. (D and E) The expression of Bcl-2, Bax, cleaved caspase-3, PARP-1 and MMP-13 was studied using western blotting. ${ }^{* *} \mathrm{P}<0.01$ vs. vector and ${ }^{\# \#} \mathrm{P}<0.01$ vs. pLVX-Puro-CTHRC1. CTHRC1, collagen triple helix repeat containing 1; Bcl-2, B-cell lymphoma-2; Bax, Bcl-2-associated X protein; PARP, poly ADP ribose polymerase; MMP, matrix metalloproteinase.

The exact cause of OA is not known; however, the degradation of extracellular matrix components is associated with elevated levels of the pro-inflammatory cytokine IL-1 $\beta$ (18). In the present study, IL-1 $\beta$ was introduced in rat chondrocytes to establish an in vitro osteoarthritis model. CTHRC1 protein expression was increased in chondrocytes in a dose-dependent manner in response to IL-1 $\beta(5,10$ and $20 \mathrm{ng} / \mathrm{ml}$; P<0.01; Fig. 2B). Furthermore, chondrocyte proliferation was suppressed by IL-1 $\beta$ in a dose-dependent manner ( $\mathrm{P}<0.01$; Fig. 2C).
CTHRC1 upregulation activates the JNK1/2 pathway in chondrocytes. The pLVX-Puro-CTHRC1 vector was constructed to and transfected into chondrocytes to induce overexpression of CTHRC1. Levels of CTHRC1 mRNA and protein were studied using RT-qPCR and western blot analysis, respectively. The expression of CTHRC1 mRNA and protein was significantly increased in chondrocytes transfected with the pLVX-Puro-CTHRC1 vector compared with chondrocytes transfected with the empty pLVX-Puro vector $(\mathrm{P}<0.01$; Fig. $3 \mathrm{~A}-\mathrm{C})$. Furthermore, CTHRC1 

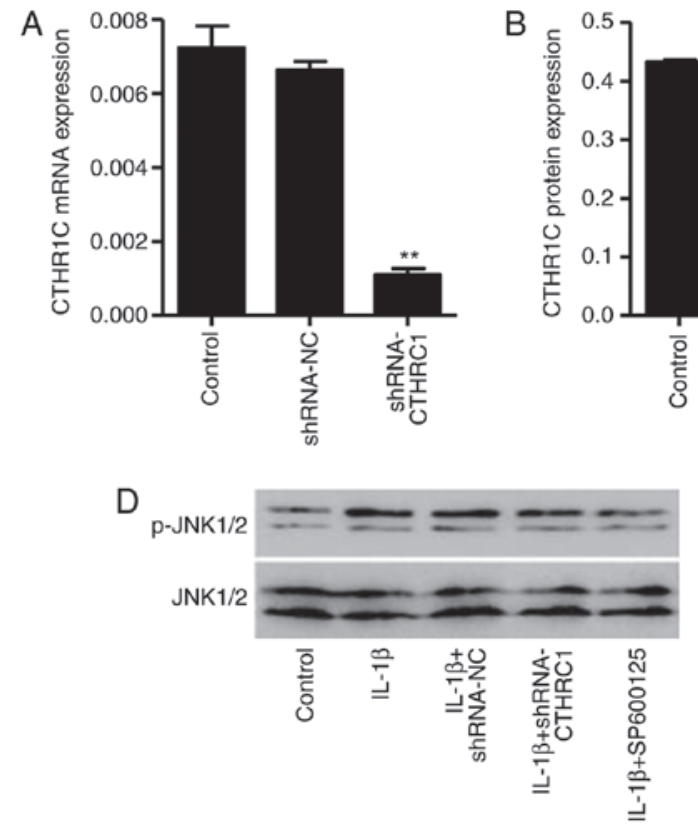
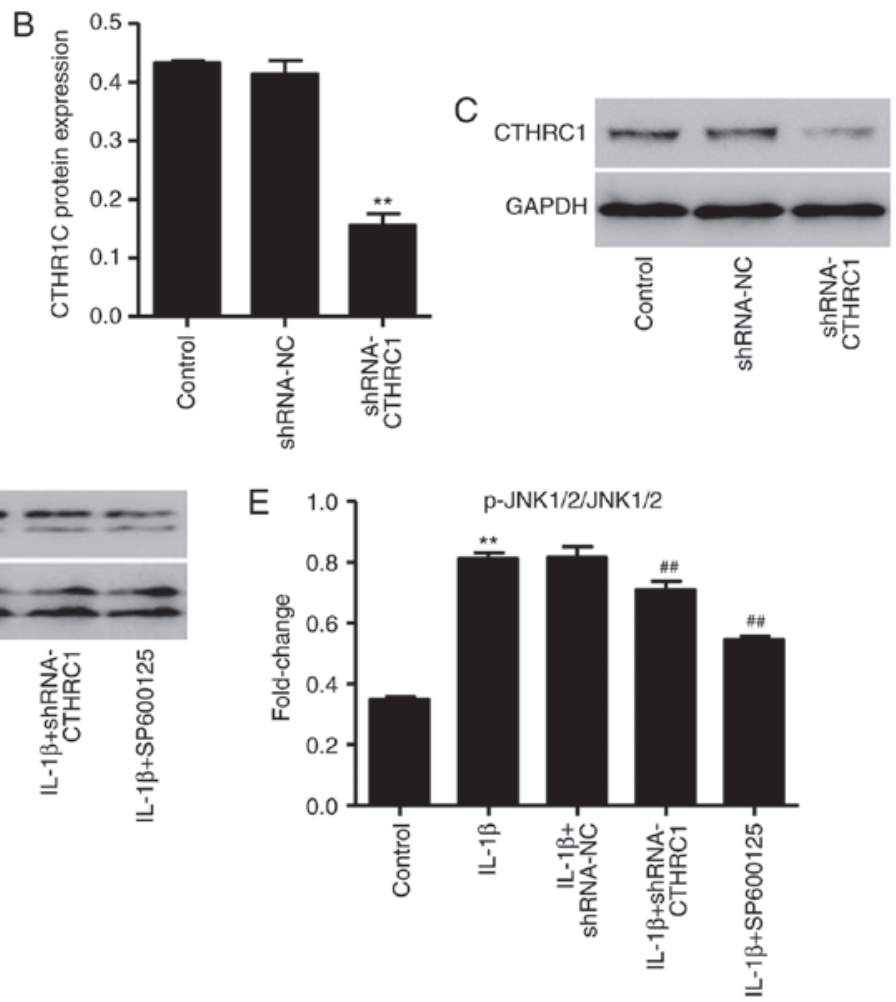

Figure 5. CTHRC1 downregulation inhibits JNK1/2 activation. IL-1 $\beta$-induced chondrocytes pLKO.1-CTHRC1-shRNA were transfected for 24 h and CTHRC1 expression was assessed using (A) reverse transcription-quantitative polymerase chain reaction and (B and C) western blotting. (D and E) The expression of p-JNK1/2 and JNK1/2 in IL-1 $\beta$-induced chondrocytes following shRNA-CTHRC1 transfection or SP600125 treatment was assessed using western blotting. ${ }^{* * *} \mathrm{P}<0.01$ vs. control and ${ }^{\# \#} \mathrm{P}<0.01$ vs. IL-1 $\beta$. CTHRC1, collagen triple helix repeat containing 1; JNK, c-Jun N-terminal kinase; IL, interleukin; sh, short hairpin; NC, negative control; p, phosphorylated.

upregulation significantly activated JNK1/2, and this activation was markedly reduced by the inhibitor, SP600125 (both $\mathrm{P}<00.01$; Fig. 3D and E).

CTHRC1 upregulation induces chondrocyte apoptosis. Following transfection with pLVX-Puro-CTHRC1 for $24 \mathrm{~h}$, the percentage of apoptotic cells and caspase-3 activity were significantly increased in chondrocytes ( $\mathrm{P}<0.01$; Fig. 4A-C); however, apoptosis and caspase-3 activity were significantly decreased following SP600125 treatment $(\mathrm{P}<0.01$; Fig. 4B and $\mathrm{C})$. The expression of MMP-13, Bcl-2, Bax, PARP-1 and cleaved caspase-3 was also measured using western blotting (Fig. 4D). The results revealed that CTHRC1 upregulation significantly increased the expression of MMP-13, Bax, PARP-1 and cleaved caspase-3 ( $<<0.01$; Fig. $4 \mathrm{E})$, whereas it significantly decreased Bcl-2 expression $(\mathrm{P}<0.01$; Fig. 4E) compared with cells transfected with the empty vector. However, SP600125 treatment significantly decreased the expression of MMP-13, Bax, PARP-1 and cleaved caspase-3 and increased Bcl-2 expression in chondrocytes with pLVX-Puro-CTHRC1 transfection ( $\mathrm{P}<0.01$; Fig. 4E), suggesting that JNK1/2 signaling is associated with the mechanism of CTHRC1 upregulation in chondrocyte apoptosis.

CTHRC1 downregulation suppresses $I L-1 \beta$-induced JNK $1 / 2$ activation. As CTHRC1 protein expression is significantly increased in patients with $\mathrm{OA}$ and in osteoarthritic chondrocytes, it was hypothesized that CTHRC1 may be correlated with OA development and chondrocyte apoptosis. The
pLKO.1-CTHRC1-shRNA vector was synthesized to downregulate the expression of CTHRC1, and the results indicated that, in chondrocytes transfected with the pLKO.1-CTHRC1-shRNA, CTHRC1 was significantly downregulated compared with control cells $(\mathrm{P}<0.01$; Fig. 5A-C). In addition, treatment with $10 \mathrm{ng} / \mathrm{ml}$ of IL-1 $\beta$ for $6 \mathrm{~h}$ significantly increased the expression of p-JNK1/2/JNK1/2 (P<0.01; Fig. 5D and E); however, this was significantly decreased following pLKO.1-CTHRC1-shRNA transfection $(\mathrm{P}<0.01$; Fig. 5D and $\mathrm{E})$. These results suggest that CTHRC1 downregulation inhibits JNK1/2 activation in IL-1 $\beta$-induced rat chondrocytes.

CTHRC1 downregulation suppresses the IL-1 $\beta$-induced apoptosis of chondrocytes. To investigate the role of CTHRC1 downregulation in chondrocytes apoptosis, apoptotic cells were assessed using flow cytometry following treatment with $10 \mathrm{ng} / \mathrm{ml}$ of IL-1 $\beta$ for $24 \mathrm{~h}$. The percentage of apoptotic cells was significantly increased compared with the control group $(\mathrm{P}<0.01$; Fig. $6 \mathrm{~A}$ and $\mathrm{B})$. However, transfection with pLKO.1-CTHRC1-shRNA significantly attenuated this increase $(\mathrm{P}<0.05$; Fig. $6 \mathrm{~A}$ and $\mathrm{B})$. To further explore the effect of JNK1/2 signaling in CTHRC1 downregulation-mediated protection against IL-1 $\beta$-induced chondrocyte apoptosis, the JNK1/2 inhibitor SP600125 $(10 \mu \mathrm{M})$ was added 30 min prior to IL-1 $\beta$. SP600125 also markedly inhibited chondrocyte apoptosis in response to IL-1 $\beta$ (Fig. 6A and B; P<0.01vs. IL-1 $\beta$ treatment alone).

To explore the role of CTHRC1 downregulation-mediated protection against IL-1 $\beta$-induced chondrocyte apoptosis, 

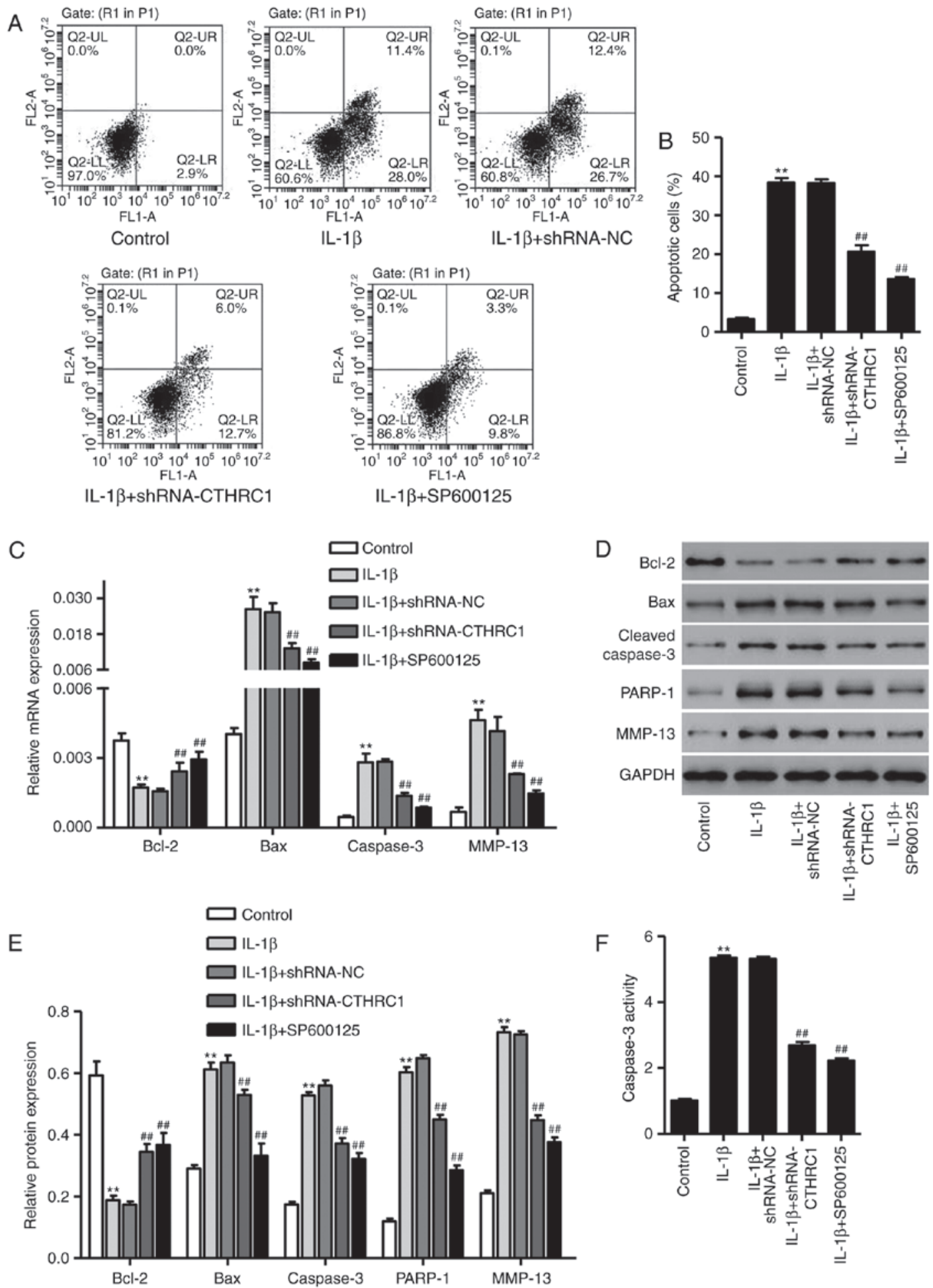

Figure 6. CTHRC1 downregulation inhibits chondrocyte apoptosis. (A) Chondrocyte apoptosis was assessed by flow cytometry and (B) statistically analyzed. (C, D and E) The expression of Bcl-2, Bax, cleaved caspase-3, PARP-1 and MMP-13 was assessed using reverse transcription-quantitative polymerase chain reaction and western blotting. (F) Changes in caspase- 3 activity were measured by spectrophotometry. ${ }^{* *} \mathrm{P}<0.01$ vs. control and ${ }^{\# \#} \mathrm{P}<0.01 \mathrm{vs}$. IL-1 3 . CTHRC1, collagen triple helix repeat containing 1; Bcl-2, B-cell lymphoma-2; Bax, Bcl-2-associated X protein; PARP, poly ADP ribose polymerase; MMP, matrix metalloproteinase.

the expressions of MMP-13, Bcl-2, Bax and cleaved caspase-3 were detected via RT-qPCR and western blotting. Compared with control cells, expression of Bcl-2 was significantly reduced $(\mathrm{P}<0.01$; Fig. 6-E), whereas the expression of Bax, PARP-1, cleaved caspase-3 and MMP-13 was significantly increased in the IL- $1 \beta$ group compared with the control $(\mathrm{P}<0.01$; Fig. 6C-E). When chondrocytes were transfected with pLKO.1-CTHRC1-shRNA or SP600125 was added prior to IL-1 $\beta, \mathrm{Bcl}-2$ expression was significantly higher compared with the IL-1 $\beta$ group $(\mathrm{P}<0.01$; Fig. $6 \mathrm{C}-\mathrm{E})$; however, MMP-13, Bax, PARP-1 and cleaved caspase- 3 expression were significantly lower $(\mathrm{P}<0.01$; Fig. 6C-E). Furthermore, 
pLKO.1-CTHRC1-shRNA or SP600125 treatment also significantly inhibited IL-1 $\beta$-induced caspase-3 activation $(\mathrm{P}<0.01$; Fig. $6 \mathrm{~F})$, suggesting that it may have a protective effect on chondrocyte apoptosis.

\section{Discussion}

$\mathrm{OA}$ is a degenerative joint disorder with multifactorial risk factors, including genetic and epigenetic factors, age, sex, ethnicity and obesity $(7,19)$. CTHRC1 protein is expressed in a number of embryonic and neonatal tissues, including developing cartilage and bone (20). In a previous study, CTHRC1 was reported to stimulate bone formation in vitro and it was suggested that the endogenous expression of CTHRC1 contributes to effective osteogenic differentiation by affecting cell proliferation and increasing the expression of osteogenic marker genes (21). CTHRC1 is associated with the severity of murine collagen antibody-induced arthritis (22) and inhibition of osteoclast differentiation (23). CTHRC1 is upregulated in patients with OA (15), suggesting a correlation between CTHRC1 and arthritis progression. The results of the present study demonstrate that CTHRC1 is more highly expressed in the joint fluid of patients with OA compared with normal joint fluid samples. However, the molecular mechanisms of and the role of CTHRC1 in human chondrocytes and OA progression remain to be elucidated.

IL-1 $\beta$ has been implicated in chondrocyte apoptosis and the degeneration of articular cartilage (24) and is of great importance for the mechanisms of degeneration and degradation of articular cartilage in OA (25); as such, IL-1 $\beta$ was used in the present study to determine the function of CTHRC1 in OA. It has previously been reported that IL- $1 \beta$ levels are increased in the synovial fluid of patients with OA and this is associated with chondrocyte apoptosis, resulting in cartilage destruction and pain $(26,27)$, which is consistent with the findings of the present study. Karaliotas et al (28) reported that the ratio of $\mathrm{Bax} / \mathrm{Bcl}-2$ was increased in patients with OA compared with a normal cartilage control group, suggesting apoptosis induction. Furthermore, caspase-3 and MMP-13, which is commonly used as a marker of chondrocyte apoptosis and matrix degradation (29), were downregulated when CTHRC1 levels were reduced. This indicates the potential role of CTHRC1 downregulation in IL-1 $\beta$-induced apoptosis inhibition, suggesting that the suppression of chondrocyte apoptosis may, in part, result in increased chondrocyte proliferation.

To investigate the underlying mechanisms of CTHRC1 downregulation, its effect on JNK1/2 activation in chondrocytes was assessed. A previous study also provided evidence that the JNK1/2 signaling pathway is of great of importance for regulating cell apoptotic signals in a number of cells (30) and is activated following IL- $1 \beta$ stimulation in osteoarthritic cartilage but not in normal cartilage (31). These studies serve to increase our understanding of the molecular mechanisms of chondrocyte proliferation, differentiation and apoptosis, and are in agreement with the in vitro experiments performed in the present study. Furthermore, JNK1/2 inhibitor SP600125 treatment and CTHRC1 downregulation were demonstrated to inhibit IL-1 $\beta$-induced JNK1/2 activation, whereas CTHRC1 upregulation mimics the effect of IL-1 $\beta$ on chondrocyte apoptosis and JNK1/2 activation, suggesting that JNK1/2 signaling is associated with CTHRC1-mediated chondrocyte proliferation and apoptosis.

The results of the present study suggest that CTHRC1 downregulation promotes chondrocyte proliferation and inhibits apoptosis by directly regulating the JNK1/2 signaling pathway in IL-1 $\beta$-induced primary rat chondrocytes. CTHRC1 upregulation may strengthen disease progression in an in vitro rat model of OA. CTHRC1 may serve as a novel therapeutic target for the regeneration of cartilage in patients with OA.

\section{Competing interests}

The authors declare that they have no competing interests.

\section{References}

1. Vos T, Flaxman AD, Naghavi M, Lozano R, Michaud C, Ezzati M, Shibuya K, Salomon JA, Abdalla S, Aboyans V, et al: Years lived with disability (YLDs) for 1160 sequelae of 289 diseases and injuries 1990-2010: A systematic analysis for the Global Burden of Disease Study 2010. Lancet 380: 2163-2196, 2012.

2. Karsdal MA, Michaelis M, Ladel C, Siebuhr AS, Bihlet AR, Andersen JR, Guehring H, Christiansen C, Bay-Jensen AC and Kraus VB: Disease-modifying treatments for osteoarthritis (DMOADs) of the knee and hip: Lessons learned from failures and opportunities for the future. Osteoarthritis Cartilage 24: 2013-2021, 2016.

3. Findlay DM and Atkins GJ: Osteoblast-chondrocyte interactionsin osteoarthritis. Curr Osteoporos Rep 12: 127-134, 2014.

4. Gore M, Tai KS, Sadosky A, Leslie D and Stacey BR: Clinical comorbidities, treatment patterns, and direct medical costs of patients with osteoarthritis inusual care: A retrospective claims database analysis. J Med Econ 14: 497-507, 2011.

5. Portal-Núñez S, Esbrit P, Alcaraz MJ and Largo R: Oxidative stress, autophagy, epigenetic changes and regulation by miRNAs as potential therapeutic targets in osteoarthritis. Biochem Pharmacol 108: 1-10, 2016.

6. Goldring MB and Goldring SR: Osteoarthritis. J Cell Physiol 213: 626-634, 2007

7. Charlier E, Relic B, Deroyer C, Malaise O, Neuville S, Collée J, Malaise MG and De Seny D: Insights on molecular mechanisms of chondrocytes death in osteoarthritis. Int J Mol Sci 17: E2146, 2016.

8. Yan S, Wang M, Zhao J, Zhang H, Zhou C, Jin L, Zhang Y, Qiu X, Ma B and Fan Q: MicroRNA-34a affects chondrocyte apoptosis and proliferation by targeting the SIRT1/p53 signaling pathway during the pathogenesis of osteoarthritis. Int J Mol Med 38: 201-209, 2016.

9. Xu XX, Zhang XH, Diao Y and Huang YX: Achyranthes bidentate saponins protect rat articular chondrocytes against interleukin-1 $\beta$-induced inflammation and apoptosis in Svitro. Kaohsiung J Med Sci 33: 62-68, 2017.

10. Wuelling $\mathrm{M}$ and Vortkamp A: Chondrocyte proliferation and differentiation. Endocr Dev 21: 1-11, 2011.

11. Shakibaei M, Allaway D, Nebrich S and Mobasheri A: Botanical extracts from Rosehip (Rosa canina), Willow Bark (Salix alba), and Nettle Leaf (Urtica dioica) suppress IL-1 $\beta$-induced $\mathrm{NF}-\kappa \mathrm{B}$ activation in canine articular chondrocytes. Evid Based Complement Alternat Med 2012: 509383, 2012.

12. Kong D, Zheng T, Zhang M, Wang D, Du S, Li X, Fang J and Cao X: Static mechanical stress induces apoptosis in rat end plate chondrocytes through MAPK and mitochondria-dependent caspase activation signaling pathways. PLoS One 8: e69403, 2013.

13. Boileau C, Pelletier JP, Tardif G, Fahmi H, Laufer S, Lavigne M and Martel-Pelletier J: The regulation of human MMP-13 by licofelone, an inhibitor of cyclo-oxygenases and5-lipoxygenase, in human osteoarthritic chondrocytes is mediated by the inhibition of the p38MAP kinase signalling pathway. Ann Rheum Dis 64: 891-898, 2005.

14. Aigner T, McKenna L, Zien A, Fan Z, Gebhard PM and Zimmer R: Gene expression profiling of serum-and interleukin-1beta-stimulated primary human adult articular chondrocytes-a molecular analysis based on chondrocytes isolated from one donor. Cytokine 31: 227-240, 2005. 
15. Rao ZT, Wang SQ and Wang JQ: Exploring the osteoarthritisrelated genes by gene expression analysis. Eur Rev Med Pharmacol Sci 18: 3056-3062, 2014.

16. Livak KJ and Schmittgen TD: Analysis of relative gene expression data using real-time quantitative PCR and the $2^{-\Delta \Delta C T}$ method. Methods 25: 402-408, 2001.

17. Yasuda H, Oh CD, Chen D, de Crombrugghe B and Kim JH: A novel regulatory mechanism of type II collagen expression via a SOX9-dependent enhancer in intron 6. J Biol Chem 292: 528-538, 2017.

18. Aigner T, Soeder S and Haag J: IL-1beta and BMPs-interactive players of cartilage matrix degradation and regeneration. Eur Cell Mater 12: 49-56, 2006.

19. Sellam J and Berenbaum F: Osteoarthritis and obesity. Rev Prat 62: 621-624, 2012 (In French).

20. Durmus T, LeClair RJ, Park KS, Terzic A, Yoon JK and Lindner V: Expression analysis of the novel gene collagen triple helix repeat containing-1 (Cthrc1). Gene Expr Patterns 6 : 935-940, 2006.

21. Kimura H, Kwan KM, Zhang Z, Deng JM, Darnay BG Behringer RR, Nakamura T, de Crombrugghe B and Akiyama $\mathrm{H}$ : Cthrcl is a positive regulator of osteoblastic bone formation. PLoS One 3: e3174, 2008

22. Kudryavtseva E, Forde TS, Pucker AD and Adarichev VA: Wnt signaling genes of murine chromosome 15 are involved in sex-affected pathways of inflammatory arthritis. Arthritis Rheum 64: 1057-1068, 2012.

23. Lawrence T: The nuclear factor NF-kappaB pathway in inflammation. Cold Spring Harb Perspect Biol 1: a001651, 2009.
24. Kapoor M, Martelpelletier J, Lajeunesse D, Pelletier JP and Fahmi H: Role of proinflammatory cytokines in the pathophysiology of osteoarthritis. Nat Rev Rheumatol 7: 33-42, 2011.

25. Bian Q, Wang YJ, Liu SF and Li YP: Osteoarthritis: Genetic factors, animal models, mechanisms, and therapies. Front Biosci 4: 74-100, 2012.

26. Jotanovic Z, Mihelic R, Sestan B and Dembic Z: Role of interleukin-1 inhibitors in osteoarthritis: An evidence-based review. Drugs Aging 29: 343-358, 2012.

27. Fernandes JC, Martel-Pelletier J and Pelletier JP: The role of cytokines in osteoarthritis pathophysiology. Biorheology 39: 237-246, 2002.

28. Karaliotas GI, Mavridis K, Scorilas A and Babis GC: Quantitative analysis of the Mrna expression levels of $B C L 2$ and $B A X$ genes in human osteoarthritis and normal articular cartilage: An investigation into their differential expression. Mol Med Rep 12: 4514-4521, 2015.

29. Cheng W, Wu D, Zuo Q, Wang Z and Fan W: Ginsenoside Rb1 prevents interleukin-1beta induced inflammation and apoptosis in human articular chondrocytes. Int Orthop 37: 2065-2070, 2013.

30. Yang D, Guo S, Zhang T and Li H: Hypothermia attenuatesis chemia/reperfusion-induced endothelial cell apoptosis via alterations in apoptotic pathways and JNK signaling. FEBS Lett 583: 2500-2506, 2009.

31. Fan Z, Söder S, Oehler S, Fundel K and Aigner T: Activation of interleukin-1 signaling cascades in normal and osteoarthritic articular cartilage. Am J Pathol 171: 938-946, 2007. 